\title{
Use of egg yolk phospholipids to generate chicken meat odorants
}

\section{Article}

\section{Accepted Version}

Creative Commons: Attribution-Noncommercial-No Derivative Works 4.0

Chen, D.-W., Balagiannis, D. P. and Parker, J. K. (2019) Use of egg yolk phospholipids to generate chicken meat odorants. Food Chemistry, 286. pp. 71-77. ISSN 0308-8146 doi: https://doi.org/10.1016/j.foodchem.2019.01.184 Available at https://centaur.reading.ac.uk/81903/

It is advisable to refer to the publisher's version if you intend to cite from the work. See Guidance on citing.

To link to this article DOI: http://dx.doi.org/10.1016/j.foodchem.2019.01.184

Publisher: Elsevier

All outputs in CentAUR are protected by Intellectual Property Rights law, including copyright law. Copyright and IPR is retained by the creators or other copyright holders. Terms and conditions for use of this material are defined in the End User Agreement.

\section{www.reading.ac.uk/centaur}

\section{CentAUR}

Central Archive at the University of Reading

Reading's research outputs online 


\section{Use of egg yolk phospholipids to generate chicken meat odorants}

De-Wei Chen ${ }^{\mathrm{a}, \mathrm{b}, *}$, Dimitrios P. Balagiannis ${ }^{\mathrm{b}}$, Jane K. Parker ${ }^{\mathrm{b}}$

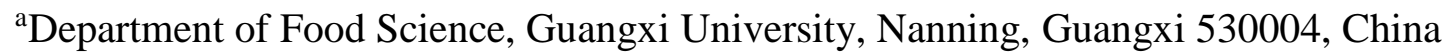

${ }^{\mathrm{b}}$ Department of Food and Nutritional Sciences, University of Reading, Whiteknights,

Reading RG6 6AP, UK

*Corresponding author: Dr. De-Wei Chen, Department of Food Science, Guangxi

University, Nanning, Guangxi 530004, China

Tel: 0086-(0)771-3237305; Fax: 0086-(0)771-3232874; E-mail: chendw@ gxu.edu.cn 


\section{Abstract}

1 Lipids, particularly phospholipids, are known to play a significant role in the

2 characteristic aroma of the different meat species. Both neutral lipids and

3 phospholipids were extracted from egg yolk and added to minced chicken $(1 \% \mathrm{w} / \mathrm{w})$

4 prior to cooking in water at $100{ }^{\circ} \mathrm{C}$ for $20 \mathrm{~min}$. Sensory analysis of the broths showed

5 that the addition of phospholipids significantly increased the chicken meat aroma

6 whereas the addition of neutral lipids did not. GC-MS analysis showed a significant

$7 \quad$ increase in most of the lipid-derived volatile components when the phospholipids

8 were added, especially 2,4-decadienal which is a characteristic odour impact

9 compound in chicken. There were very few significant changes in the volatile profile

10 when the neutral lipids were added. These data provide direct evidence that the

11 addition of phospholipids can enhance chicken meat aroma, and addition of egg yolk

12 phospholipids could be applied to improve chicken meat aroma.

14 Keywords: chicken meat; aroma; phospholipids; egg yolk; lipid-derived volatile; 


\section{Introduction}

Chicken broth in China is well known for its rich, rounded, sweet, aromatic notes, and consumers are keenly aware of the difference in flavour of slow growing natively reared chickens compared to the intensively reared chickens (broilers) which are grown much more rapidly and lack flavour. A recent report (Feng, Cai, Fu, Zheng, Xiao \& Zhao, 2018) demonstrated using GC-olfactometry and aroma extract dilution analysis that the key difference between chicken broth prepared from either native or commercially reared chickens was in the concentration of lipid-derived compounds, rather than in the Maillard or sulfur-derived volatiles.

Phospholipids are known to play a significant role in the formation of the characteristic aroma of different meat species (Mottram, 1998; Whitfield \& Mottram, 1992). In chicken, aldehydes with $>5$ carbon atoms, such as hexanal, (E)-2-nonenal, (E)-2-decenal, (Z)-2-decenal, (E,E)-2,4-decadienal, (E)-2-undecenal, (E,Z,Z)-2,4,7tridecatrienal, and also 1-octen-3-one, are generated by thermally induced oxidation and decomposition of the endogenous fatty acids. These lipid-derived compounds contribute to the characteristic chicken aroma whereas 2-methyl-3-furanthiol and other related cysteine- and ribose-derived compounds tend to provide the non-specific meaty character in meat (Jayasena, Ahn, Nam \& Jo, 2013; Mottram, 1998; Shi \& Ho, 1994; Stephan \& Steinhart, 1999). In addition, interactions between lipid oxidation products and Maillard reaction products (Farmer \& Mottram 1990; Mottram \& Whitfield, 1995; Whitfield et al., 1992) can generate thiophenes, thiazoles, furans, 
pyrazines and pyridines with alkyl substituents which are derived from lipid, leading to a modified and species specific overall aroma of cooked meat.

Egg yolk is a good source of phospholipids, and the content of phospholipids is about $10 \%$ of the wet weight of the egg yolk (Gladkowski, Chojnacka, Kielbowicz, Trziszka \& Wawrzenczyk, 2012). The fatty acid profile of egg phospholipids is similar to that of chicken meat, although the polyunsaturated fatty acids (PUFAs) in chicken meat are higher than those in the egg yolk (Fredriksson, Elwinger \& Pickova, 2006; Katz, Dugan \& Dawson, 1966). Egg phospholipids are rich in PUFAs, especially linoleic acid (C18:2), arachidonic acid (C20:4) and docosahexaenoic acid (C22:6) (Katz et al., 1966). Thus, egg yolk can be used as a source of these important precursors for the generation of key aroma compounds in chicken. For example, thermally treated egg phospholipids $\left(145^{\circ} \mathrm{C}\right.$, for $\left.20 \mathrm{~min}\right)$ have been shown to produce an abundance of key aroma compounds, such as hexanal, (E,E)-2,4-decadienal, 1-octen-3-one, trans-4,5epoxy-(E)-2-decenal, (Z)-2-decenal, (E)-2-decenal and (E)-2-undecenal (Lin \& Blank, 2003), which are important for the aroma of chicken meat.

Methods for the isolation and purification of egg yolk lipids are widely reported in the literature and the purity of phospholipids and neutral lipids fraction is quite satisfactory. Generally, egg yolk phospholipids are extracted with ethanol, and then purified by removing neutral lipids. Palacios \& Wang (2005) used a multistep extraction with ethanol and hexane, followed by addition of chilled acetone to precipitate the phospholipids in the final purification step. They isolated phospholipids with $95.9 \%$ purity, and the neutral lipid only contained $1.8 \%$ of the 
phospholipids. Gladkowski et al. (2012) used acetone at $-20{ }^{\circ} \mathrm{C}$ to precipitate and wash phospholipids, and they obtained a pure phospholipid fraction in $9.5 \%$ yield, and the high purity phospholipids contained phosphatidylcholine (78\%) and phosphatidylethanolamine (21\%).

The hypothesis of our work is that reactive precursors involved in the formation of characteristic lipid-derived compounds can be provided by addition of phospholipids, in particular egg yolk phospholipids, which have a similar composition to chicken phospholipids. Phospholipids extracted from egg yolk will be added to minced chicken breast prior to cooking in water at $100{ }^{\circ} \mathrm{C}$, mimicking the preparation of traditional Chinese chicken broth. Although egg yolk has been used as part of a complex mixture of ingredients to prepare process flavours (Tian, 2014), to the best of our knowledge, no research has been published where egg yolk phospholipids have been used specifically to increase the key volatile components of chicken aroma in a real food.

\section{Materials and methods}

\subsection{Reagents and Chemicals}

Aroma chemicals were obtained from the following suppliers: 2-furfural, 3-octen-2one, benzeneacetaldehyde, carbon disulfide and 1-decene from Fisher Scientific (Loughborough, U.K.); 1-octen-3-one from Danisco (Kettering, U.K.); benzaldehyde and 1-decanol from Givaudan (Milton Keynes, U.K.); (E,E)-2,4-decadienal from Lancaster Synthesis (Heysham, U.K.); 2-ethylfuran, 1-penten-3-one, 2,3pentanedione, (E)-2-butenal, hexenal, butanal and (E)-2-heptenal from Oxford 
Chemicals (Hartlepool, U.K.); (E,E)-2,4-nonadienal, 2,3,5-trimethylpyrazine, 2,3butanedione, decanal, dimethyl trisulfide, heptanal, hexanal, undecanal, (Z)-4heptenal, nonanal, (E)-2-nonenal, (E)-2-octenal, (E)-2-undecenal, (E,E)-2,4octadienal, 2-nonanone, tetramethylpyrazine, (E)-2-(2-pentenyl)furan, 1-pentanol, (Z)-2-penten-1-ol, (E,E)-2,4-heptadienal, 3,5-octadien-2-one, 1-octanol, 1-nonanol, 6methyl-2-heptanone, 3octanone, 2-octanone, 2,3-octanedione, methional, hydrogen sulfide, methanethiol, nonane, 1-butanol, 1-tetradecene, 3-nonen-2-one, (E)-2-octen1-ol, and 6-methyl-3,5-heptadiene-2-one from Sigma-Aldrich Ltd. (Gillingham, U.K.); 1-octen-3-ol, pentanoic acid, and propanoic acid from Synergy (High Wycombe, U.K.); Pentanal, octanal, nonanal, decanal and dodecanal from Polyscience (Cambridgeshire, U.K.); 2-pentylfuran and 3-ethylcyclopentanone from Avocado (London, U.K.); 2-methylbutanal and 3-methylbutanal from Alfa Aesar (Lancashire, U.K.); 2-pentanone, 3-hexanone, 2-heptanone, 2-nonanone, 2-decanone, 3,5-heptadien-2-one and 2-undecanone from Koch-Light (Haverhill, U.K.); dimethyl sulfide, dimethyl trisulfide and 1-hexanol from IFF(New York, USA). 1,2-

Dichlorobenzene in methanol $(130.6 \mathrm{ng} / \mu \mathrm{L})$ and alkane standard $\mathrm{C}_{5}-\mathrm{C}_{25}(100 \mathrm{ng} / \mu \mathrm{L}$ in diethyl ether), used as GC-MS standards, HPLC-grade hexane, ethanol and acetone were obtained from Sigma-Aldrich Ltd. (Gillingham, U.K.); HPLC-grade water was obtained from Fisher Scientific (Loughborough, U.K.).

\subsection{Lipid extraction}

Phospholipids extraction. The method employed was that reported by Gladkowski et al. (2012) with minor modifications. Briefly, fresh egg yolk (20 g) and $60 \mathrm{ml}$ of 
103

ethanol were mixed and stirred for $30 \mathrm{~min}$. The supernatant was removed, the extraction of egg yolk with ethanol was repeated twice and the supernatants combined. The precipitate was retained for extraction of neutral lipids. The ethanol was evaporated from the combined supernatants under reduced pressure, then the residue was dissolved in hexane $(30 \mathrm{ml})$ and placed in an ice bath $\left(0{ }^{\circ} \mathrm{C}\right)$. Next, $60 \mathrm{ml}$ of cold acetone $\left(-20^{\circ} \mathrm{C}\right)$ was added into the stirred mixture to precipitate phospholipids, and then the precipitate was washed 5 times with $20 \mathrm{ml}$ portions of cold acetone $\left(-20^{\circ} \mathrm{C}\right)$.

Neutral lipids extraction. The method employed was that reported by Palacios et al. (2005) with minor modifications. After extraction of the egg yolk with ethanol, the neutral lipids in the precipitate were extracted twice with $50 \mathrm{ml}$ of hexane, and the combined hexane layers washed four times, each with $50 \mathrm{ml}$ of $90 \%$ ethanol. Finally, the hexane was evaporated under reduced pressure, and the neutral lipids from egg yolk were obtained.

The minor residual solvents in the phospholipids and neutral lipids were removed by high vacuum at room temperature for $10 \mathrm{~h}$.

\subsection{Sample preparation}

Fresh chicken breast fillets without skin or bone were bought from a local supermarket. The chickens had been reared commercially and were of basic quality i.e. they were not specified as organic, free range or corn-fed chickens. The chicken meat $(\sim 500 \mathrm{~g})$ was ground in a domestic meat mincer (Kenwood, Havant, UK) and thoroughly mixed. The samples were prepared as follows: 
125

126

127

128

129

130

131

132

133

134

135

1) Phospholipids sample: $0.10 \mathrm{~g}$ phospholipids, $20 \mathrm{~mL}$ water.

2) Neutral lipids sample: $0.10 \mathrm{~g}$ neutral lipids, $20 \mathrm{~mL}$ water.

3) Chicken meat sample: $10.0 \mathrm{~g}$ chicken meat, $20 \mathrm{~mL}$ water.

4) Chicken meat \& neutral lipids sample: $10.0 \mathrm{~g}$ chicken meat, $0.10 \mathrm{~g}$ neutral lipids, $20 \mathrm{~mL}$ water.

5) Chicken meat \& phospholipids sample: $10.0 \mathrm{~g}$ chicken meat, $0.10 \mathrm{~g}$ phospholipids, $20 \mathrm{~mL}$ water.

Finally the samples were sealed in $100 \mathrm{~mL}$ glass Duran bottles and cooked in boiling water $\left(100^{\circ} \mathrm{C}\right)$ for $20 \mathrm{~min}$ and then cooled in an ice-bath. Each treatment was carried out in quadruplicate and all samples were prepared from the same batch of chicken mince.

\subsection{Dynamic Headspace Extraction (DHE)}

DHE was used for the extraction of the volatiles, following the method described by Methven, Tsoukka, Oruna-Concha, Parker \& Mottram (2007) with minor modifications. After cooking, the entire contents of each Duran bottle was mixed with sodium chloride $(15 \mathrm{~g})$ and HPLC grade water $(5 \mathrm{~mL})$ and placed in a $250 \mathrm{~mL}$ conical flask fitted with a Dreschel head. The flask was incubated in a water bath at $50{ }^{\circ} \mathrm{C}$, and the volatiles in the headspace were swept onto Tenax absorbent using a flow of nitrogen ( $40 \mathrm{~mL} / \mathrm{min}$ ) for $60 \mathrm{~min}$. After sweeping, $1.0 \mu \mathrm{L}$ of 1,2-dichlorobenzene in methanol $(130.6 \mathrm{ng} / \mu \mathrm{L})$ was added as an internal standard to the trap, followed by a purge of $100 \mathrm{~mL} / \mathrm{min}$ for $10 \mathrm{~min}$ to remove excess solvent and moisture.

\subsection{GC-MS Analysis of Volatile Compounds}


147

The DHE samples were analysed using Agilent 7890A-5975 GC-MS system (Agilent Technologies Co. Ltd., Palo Alto, CA, USA) equipped with an automated thermal desorber (Turbomatrix ATD), using a Supelcowax 10 column $(60 \mathrm{~m} \times 0.25 \mathrm{~mm}$ i.d., $0.5 \mu \mathrm{m}$ film thickness, from Sigma, Poole, UK) and a DB 5 column $(60 \mathrm{~m} \times 0.25 \mathrm{~mm}$ i.d., $1 \mu \mathrm{m}$ film thickness from J\&W Scientific, Agilent, Palo Alto, CA, USA) under instrumental conditions described by Methven et al. (2007). The identification of the compounds was based on the comparison of their mass spectra with spectra from the NIST 11 Mass Spectral Database (NIST/EPA/MSDC, 1992). The linear retention index (LRI) was calculated for each volatile using the retention times of a series of $\mathrm{C}_{5}-\mathrm{C}_{25}$ n-alkanes. The identities of most of the volatiles were confirmed if their mass spectra and LRI matched those of authentic compounds run under the same analytical conditions in our laboratory. Volatiles were considered as tentatively identified by matching their mass spectra with the references mass spectra in the NIST mass spectral library, and by comparison of their LRI to the NIST database (NIST Chemistry WebBook, 2017). Volatiles were semi-quantitatively determined by comparison of the peak areas against those of the internal standard using a response factor of 1 for each compound.

\subsection{Quantitative descriptive analysis (QDA)}

The aroma of the three chicken samples was assessed by QDA. The solids were removed from the three chicken samples and the clear liquids $(10 \mathrm{~g})$ were put in brown glass containers with caps. The containers were kept in a water bath at $50{ }^{\circ} \mathrm{C}$ for 20 min to ensure the accumulation of volatiles in the headspace. Prior to the 
169

170

171

172

173

174

175

analysis, 9 panellists (male $=4$, female $=5$ ), all of whom had previous experience in QDA, attended a number of round table discussions for the descriptive analysis where samples and references were presented. The panel reached a consensus on the following odor attributes ('chicken broth', 'chicken meat', 'cooked vegetable', 'oily', 'roasted' and 'sulfur') which they used to describe the sensory characteristics of the three chicken samples. The panellists did not perceive a rancid or fatty off-flavour in any of the samples, but used the term oily to describe a fresh oily note. For the scoring sessions, the samples labelled with random three-digit codes were presented in ventilated tasting booths illuminated with white light. The panel members individually evaluated the odor qualities by sniffing samples, and quantified the attributes using an unstructured line scale (scaled 0-100). All samples were assessed in duplicate by each assessor. The data were collected using Compusense 5 software (Compusense Inc., Guelph, Ontario, Canada).

\subsection{Statistical Analysis}

The GC-MS data were analysed using one-way analysis of variance (ANOVA) and means were compared using the Fisher's least significant difference (LSD) test at $\mathrm{P}=$ 0.05. SENPAQ version 3.2 (Qi Statistics, Reading, U.K.) was used to carry out twoway ANOVA and Tukey's HSD at alpha $=0.05$ on the sensory data. Principal component analysis (PCA) using XLSTAT was carried out on the sensory data with the volatile compounds added as supplementary variables.

\section{Results and Discussion}

\subsection{Sensory evaluation}


191

192

The sensory profiles of the three chicken samples are shown in Figure 1. All the samples were scored highly for the 'chicken meat' and 'chicken broth' attributes, whereas the attributes of 'oily', 'roasted' and 'sulfury' received much lower mean scores. The score for the 'chicken broth' attribute in the chicken heated with neutral lipids was significantly higher than for the samples of chicken cooked with the phospholipids ( $\mathrm{p}=0.004)$, whereas the scores for both the 'chicken meat' attribute and the 'roasted' attribute were significantly higher for the chicken cooked with phospholipids compared to the other two samples ( $\mathrm{p}=0.018$ and 0.020 respectively). It is interesting that having added phospholipids to the sample, the term chosen by the panel to describe the aroma was 'chicken meat' rather than a fatty term.

\subsection{The origin and aroma characteristic of lipid-derived volatiles.}

The volatiles in Table 1 were classified according to their possible origin. The formation of the characteristic aroma compounds of chicken meat (E,E)-2,4decadienal (fatty, fried), and others such as 2-nonenal (fatty, fried, fatty, green), 1octen-3-ol (mouldy, mushroom-like), 1-octen-3-one (mouldy, mushroom-like) and (E,E)-2,4-nonadienal (fatty, fried, green) are formed from the autoxidation of $\omega-6$ fatty acids such as linoleate and arachidonate, while (E)-2-undecenal (fatty, green), (E)-2-decenal (fatty, fried), decanal (aldehydic, waxy), octanal (aldehydic, waxy) and nonanal (aldehydic, waxy) originate from the autoxidation of $\omega-9$ fatty acids such as oleate. 2,4-Heptadienal (fatty, green) and 3,5-octadien-2-one (fruity, fatty) originate from $\omega$-3 fatty acids such as linolenate (Hsieh \& Kinsella, 1989; Kawai, 1996; Shi et al., 1994; Wurzenberger \& Grosch, 1984; Zamora, Navarro, Aguilar \& Hidalgo, 2015; 
213

214

Zhou, Zhao, Bindler \& Marchioni, 2014). 2-(2-Pentenyl)furan (beany, green, buttery, painty, metallic) and 2-pentylfuran (green, beany, earthy, metallic) are known to be mainly responsible for the undesirable reversion flavour of soybean oil, and are formed from the $\mathrm{C} 10$ hydroperoxide of linolenate and linoleate respectively by the singlet oxygen oxidation (Smagula, Ho \& Chang, 1979).

\subsection{Comparison of lipid samples.}

Since the release of aroma compounds is very different from an aqueous meat mix than it is from the extracted lipid fractions, the two sets of samples will be discussed separately. Overall, the headspace of the heated phospholipid sample was significantly richer in number and abundance of lipid-derived volatiles compared to that of the neutral lipid sample as shown in Table 1. The compounds derived from the more reactive $\omega-3$ and $\omega-6$ fatty acids were all significantly higher in the phospholipid sample. Interestingly, some of the compounds derived from the less reactive $\omega-9$ fatty acids also increased, in particular 2-undecenal, as did 6-methyl-3,5-heptadiene-2-one, an oxidative breakdown product of carotenoids. It has been reported previously (Elmore, Mottram, Enser \& Wood, 1999) that once the lipid oxidation process has been initiated by the more reactive, more unsaturated fatty acids, this promotes the oxidation of the less reactive fatty acids. This is also evident from the increase in methylketones which are breakdown products of saturated fatty acids. 1-Tetradecene was the exception as it was found to be significantly higher in the neutral lipids compared to the phospholipids.

The presence of Maillard reaction products in the heated lipid samples is surprising, 
but we can only assume that these were formed from low levels of precursors which were co-extracted along with the lipids. The more polar solvent used to extract the phospholipids is consistent with there being more Maillard reaction precursors present, and therefore more Maillard reaction products in the phospholipids. It is also consistent with the work of Hidalgo \& Zamora (2004 and 2016) who have shown that products of lipid oxidation can facilitate the degradation of amino acids to their corresponding Strecker aldehydes. This can explain the increase in 2- and 3methylbutanal in the heated phospholipid sample. Products of the Maillard reaction have been reported before in heated phospholipids (Stephan et al., 1999).

Both hexanal and 2,4-decadienal are often used as primary marker compounds of the oxidation of $\omega-6$ fatty acids (Choe \& Min, 2006). They were 12 times and 100 times higher in the phospholipid compared to the neutral lipids, respectively, confirming that egg yolk phospholipids are more oxidatively sensitive than egg yolk neutral lipids under the present experimental conditions. Phosphatidylcholines, particularly those still bound up in the cell membrane, are initially more resistant to thermal oxidation compared to their corresponding triglycerides, however, Zhou et al. (2014) showed that phosphatidylcholine produces over 5 times more unsaturated carbonyls than triglycerides do. Phospholipids have both hydrophilic and hydrophobic groups in the same molecule, so they are good emulsifiers, they decrease the surface tension of the matrix and increase the diffusion rate of oxygen from the surface to the interior thereby accelerating lipid oxidation in an oil matrix. In the present study, the added phospholipids were homo-dispersed in the meat matrix, so they had a much more 
larger surface area than the hydrophobic neutral lipids. Furthermore, phospholipids have a negative charge that attracts prooxidant metals to accelerate oxidation. They also contain a higher proportion of PUFAs (Choe et al., 2006; Cui \& Decker, 2016; Min \& Ahn, 2005; Reis \& Spickett, 2012). As shown in Table 2, the PUFAs in the phospholipids are higher than those in the triglycerides. As PUFAs are more prone to oxidation (Choe et al., 2006; Min et al., 2005), more volatiles were generated when the phospholipid samples were cooked. It has been reported that egg yolk phospholipids can have good antioxidative activity (Cui et al., 2016), and that the antioxidative activity of egg yolk phospholipids decreased with an increase in the degree of saturation of fatty acid chains within the phospholipids (Sugino et al., 1997), but we see no evidence of antioxidant activity in our system.

\subsection{Comparison of chicken samples with added lipids.}

The trends in volatile compounds in the three chicken samples were consistent with those already discussed for the lipid samples. All but two $\omega-3$ and $\omega-6$ derived compounds were significantly higher in the chicken sample containing phospholipids compared to the chicken alone, and in most cases there was no significant difference between the chicken alone and the chicken cooked with neutral lipids. There was a similar trend for some of the $\omega-9$ derived compounds, but nonanal, 1-decene, and decanol were all significantly higher in the chicken cooked with neutral lipids. The Maillard reaction products tended to show no significant difference between samples, although the two Strecker aldehydes, 2- and 3-methylbutanal, both significantly increased when the lipids were included, particularly the phospholipids. Lipid 
degradation products have been shown to undergo a Strecker-type degradation

(Hidalgo et al., 2004 and 2016). The sulfur containing compounds had a high standard deviation associated with them, as is often the case, and did not show any significant differences between samples.

Linoleic acid is the predominant PUFA in both the phospholipids and neutral lipids of chicken meat and egg yolk. In phospholipids, the most favoured position for formation of hydroperoxides during the radical initiation step of autoxidation is at the C9 position (Reis et al., 2012). In triglycerides, or the corresponding methyl esters, the hydroperoxides are formed at both C9 and C13 position (Choe et al., 2006; Ho \& Chen, 1994). The C9 hydroperoxide is the precursor for 2,4-decadienal whereas the C13 hydroperoxide is the precursor for hexanal. So linoleate residues present in triglycerides can produce both (E,E)-2,4-decadienal and hexanal whereas when the same residue is assembled in a polar phospholipid, 2,4-decadienal is the major product, explaining why phospholipids produce (E,E)-2,4-decadienal more effectively than neutral lipids.

The ratios of (E,E)-2,4-decadienal to hexanal in the neutral lipid sample and phospholipid sample are 0.087 and 0.73 , respectively, showing clearly that phospholipids generate 2,4-decadienal far more effectively than neutral lipids. The ratios in the chicken sample, chicken \& neutral lipid sample and chicken \& phospholipid sample show a much diminished effect $(0.008,0.008$ and 0.011$)$. Neutral lipids had no positive effect on this ratio and the content of 2,4-decadienal, whereas the ratio for the chicken and phospholipid sample increased slightly. This apparent 
301

302

303

"loss" of 2,4-decadienal in the presence of meat can be attributed to the interaction of this highly reactive alkadienal with other components of the meat, either the reactive intermediates generated in the meat by the Maillard reaction (such as $\mathrm{H}_{2} \mathrm{~S}, \mathrm{NH}_{3}$ and reactive dicarbonyls), or to the reaction with free amino groups. Perez-Juan, Flores \& Toldra (2008) have also suggested that these compounds may get trapped within the meat. Examination of Table 1 shows that those compounds which had the greatest apparent "loss" are highly reactive 2,4-alkadienals, followed by the 2-alkenals, whereas the alkanals and alcohols were less affected.

\subsection{Correlation with sensory}

Figure 2 shows the principal component analysis carried out on the sensory data for the three chicken samples. The volatile compounds were included as supplementary variables and used to explain the differences in the sensory profile. It summarises much of the discussion above. The chicken sample containing the phospholipids is correlated with two sensory attributes which showed significant differences between the samples: 'chicken meat' and 'roasted' and also 'sulfur' (not significant). This sample, and the associated attributes, are correlated with all the $\omega-3$ and $\omega-6$ lipidderived compounds, confirming the key role of phospholipids (rather than the neutral lipids) in generating these compounds and the characteristic aroma of chicken meat. This sample is also correlated with octanol and octanal (derived from $\omega-9$ fatty acids), methylketones (derived from saturated fatty acids) and 6-methyl-3-5-hexadien-2-one (derived from carotenoids) showing that the increase in lipid degradation was across the whole range of fatty acids and even affected the carotenoids. The carotenoids are 
323

naturally occuring in chicken fat, and being non-polar are co-extracted with the lipid fractions turning them a pale orange.

Although hexanal increased in the phospholipid containing samples, it has less effect on chicken meat aroma because of its relatively high odour detection threshold (4.5 $\mu \mathrm{g} / \mathrm{kg}$ ) (Shi et al., 1994) compared to that of 2,4-decadienal $(0.07 \mu \mathrm{g} / \mathrm{kg}$ ) (Shi et al., 1994) which imparts a characteristic fatty fried chicken note. However, large quantities of hexanal can induce off-flavour (Byrne, Bredie, Mottram \& Martens, 2002). It is therefore important to note that no fatty off-flavour was found by the panellists.

Although chicken and roasted notes could arise from an increase in 2,4-decadienal (and other related compounds) the terms meat and sulfur are not generally associated with lipid degradation. These may be indicators of low levels of potent sulfur and/or Maillard-derived compounds present in the meat at levels below the detection limit of the analytical method. These compounds generally require high temperatures for their formation, so the mild cooking process would not have favoured their formation.

Furthermore, the meaty character could be generated by the interaction between the lipid degradation products and $\mathrm{H}_{2} \mathrm{~S}$ derived from the breakdown of cysteine to produce subthreshold levels of potent sulfur compounds. This is currently under further investigation.

The 'chicken broth' note associated with the neutral lipids sample is likely to represent the underlying aroma before the introduction of the phospholipids. Table 1 shows that potent compounds such as butanedione, methional, methanethiol, dimethyl 
345

sulfide, dimethyl disulfide and dimethyl trisulfide were all present in the chicken and chicken with neutral lipid samples. Because of the potato and vegetable aroma of all but butanedione, it is very likely that these compounds contributed to a more brothy note. These compounds did not increase significantly when the phospholipids were added, and it is likely that the roasty, chicken meat and sulfur aroma generated from the phospholipids masked the chicken broth notes. Under these processing conditions, we were unable to detect the characteristic 2-methyl-3-furanthiol and related compounds which impart a typical meaty brothy note. In practical applications, the additional use of ribose (or xylose) as well as egg yolk, egg yolk phospholipids or egg-lecithin might further increase the 'chicken meat' aroma (Aliani \& Farmer, 2005; Mottram et al., 1995).

\section{Conclusion}

Clearly, it has been demonstrated, both instrumentally and sensorially, that egg yolk phospholipids, rather than egg yolk neutral lipids, increase the formation of characteristic aroma compounds in chicken meat samples. Addition of egg yolk phospholipids can be applied to improve chicken meat aroma in the food industry.

\section{Acknowledgements}

This study was supported by the China Scholarship Council (No. 201706665009). The authors wish to acknowledge Huiqi Yeo for helping with the samples analysis.

\section{Conflict of interest}

There is no conflict of interest about this article. 


\section{References}

Aliani, M., \& Farmer, L. J. (2005). Precursors of chicken flavor. II. Identification of key flavor precursors using sensory methods. J. Agric. Food Chem., 53(16), 64556462.

Byrne, D. V., Bredie, W., Mottram, D. S., \& Martens, M. (2002). Sensory and chemical investigations on the effect of oven cooking on warmed-over flavour development in chicken meat. Meat Sci., 61, 127-139.

Choe, E., \& Min, D. B. (2006). Mechanisms and factors for edible oil oxidation. Compr. Rev. in Food Sci.F., 5(4), 169-186.

Cui, L., \& Decker, E. A. (2016). Phospholipids in foods: prooxidants or antioxidants? J. Sci. Food Agric., 96(1), 18-31.

Elmore, J. S., Mottram, D. S., Enser, M., \& Wood, J. D. (1999). Effect of the polyunsaturated fatty acid composition of beef muscle on the profile of aroma volatiles. J. Agric. Food Chem., 47(4), 1619-1625.

Farmer, L. J. and Mottram D. S. (1990). Interaction of lipid in the Maillard reaction between cysteine and ribose: the effect of a triglyceride and three phospholipids on the volatile products. J. Sci. Food Agric., 53(4), 505-525.

Feng, Y., Cai, Y., Fu, X., Zheng, L., Xiao, Z., \& Zhao, M. (2018). Comparison of aroma-active compounds in broiler broth and native chicken broth by aroma extract dilution analysis (AEDA), odor activity value (OAV) and omission experiment. Food Chem., 265, 274-280.

Fredriksson, S., Elwinger, K., \& Pickova, J. (2006). Fatty acid and carotenoid 
composition of egg yolk as an effect of microalgae addition to feed formula for laying hens. Food Chem., 99(3), 530-537.

Gladkowski, W., Chojnacka, A., Kielbowicz, G., Trziszka, T., \& Wawrzenczyk, C. (2012). isolation of pure phospholipid fraction from egg yolk. J. Am. Oil Chem. Soc., 89(1), 179-182.

Hidalgo, F. J., \& Zamora, R. (2004). Strecker-type degradation produced by the lipid oxidation products 4,5-epoxy-2-alkenals. J. Agric. Food Chem., 52(23), 71267131.

Hidalgo, F. J., \& Zamora, R. (2016). Amino acid degradations produced by lipid

Kawai, T. (1996). Fish flavor. Crit. Rev. Food Sci. Nutr., 36(3), 257-298. 
Methven, L., Tsoukka, M., Oruna-Concha, M. J., Parker, J. K., \& Mottram, D. S. (2007). Influence of sulfur amino acids on the volatile and nonvolatile components of cooked salmon (Salmo salar). J. Agric. Food Chem., 55(4), 1427-1436.

Min, B., \& Ahn, D. U. (2005). Mechanism of lipid peroxidation in meat and meat products - A review. Food Sci. Biotechnol., 14(1), 152-163.

Mottram, D. S. (1998). Flavour formation in meat and meat products: a review. Food

Mottram, D. S. and Whitfield, F. B. (1995). Volatile compounds from the reaction of Biomembranes, 1818, 2374-2387. 
432

433

Shi, H., \& Ho, C. T. (1994). The flavour of poultry meat. In: Shahidi, F. (Ed.), Flavor of meat and meat products (pp. 52-70): Springer, Boston, MA.

Smagula, M. S., Ho, C. T., \& Chang, S. S. (1979). The synthesis of 2-(2-pentenyl) furans and their relationship to the reversion flavor of soybean oil. J. Am. Oil Chem. Soc., 56(4), 516-519.

Stephan, A., \& Steinhart, H. (1999). Identification of character impact odorants of different soybean lecithins. J. Agric. Food Chem., 47(7), 2854-2859.

Sugino, H., Ishikawa, M., Nitoda, T., Koketsu, M., Juneja, L. R., Kim, M., \& Yamamoto, T. (1997). Antioxidative activity of egg yolk phospholipids. J. Agric. Food Chem., 45(3), 551-554.

Tian, J. (2014). Cooked chicken flavor essence and its preparation method. CN103976336A Tianjin Chunfa Biotechnology Group Co., Ltd., Peop. Rep. China.

Whitfield, F. B., \& Mottram, D. S. (1992). Volatiles from interactions of Maillard reactions and lipids. Crit. Rev. Food Sci. Nutr., 31(1-2), 1-58.

Wurzenberger, M. and Grosch, W. (1984). The formation of 1-octen-3-ol from the 10hydroperoxide isomer of linoleic acid by a hydroperoxide lyase in mushrooms (Psalliota bispora). BBA Lipids and Lipid Metabolism, 794(1), 25-30.

Zamora, R., Navarro, J. L., Aguilar, I., \& Hidalgo, F. J. (2015). Lipid-derived aldehyde degradation under thermal conditions. Food Chem., 174, 89-96.

Zhou, L., Zhao, M., Bindler, F., \& Marchioni, E. (2014). Comparison of the volatiles formed by oxidation of phosphatidylcholine to triglyceride in model systems. $J$. Agric. Food Chem., 62(33), 8295-8301. 
Table 1. Mean Values (approx ng/sample extraction) $(n=4)$ of the Volatile Compounds Identified in Headspace of the Heated Samples.

\begin{tabular}{|c|c|c|c|c|c|c|c|c|c|c|c|}
\hline \multirow[b]{2}{*}{ Compound Name } & \multirow[b]{2}{*}{ Code } & \multirow[b]{2}{*}{$\begin{array}{l}\text { LRI }^{1} \\
\text { DB5 }\end{array}$} & \multirow[b]{2}{*}{$\begin{array}{l}\text { LRI }^{2} \\
\text { WAX }\end{array}$} & \multirow[b]{2}{*}{$\mathrm{ID}^{3}$} & \multicolumn{3}{|c|}{$\underline{\text { Heated extracted lipids }}$} & \multicolumn{4}{|c|}{ Minced chicken heated with extracted lipids } \\
\hline & & & & & $\begin{array}{c}\text { Neutral lipids } \\
\text { mean } \pm \mathrm{SD}^{4}\end{array}$ & $\begin{array}{c}\text { Phospholipids } \\
\text { mean } \pm \mathrm{SD}^{4}\end{array}$ & $\begin{array}{l}\text { Lipid } \\
\text { Sig }^{5}\end{array}$ & $\begin{array}{l}\text { Meat alone } \\
\text { mean } \pm \mathrm{SD}^{4}\end{array}$ & $\begin{array}{l}\text { With neutral lipids } \\
\text { mean } \pm \mathrm{SD}^{4}\end{array}$ & $\begin{array}{c}\text { With phospholipids } \\
\text { mean } \pm \mathrm{SD}^{4}\end{array}$ & $\begin{array}{l}\text { Meat } \\
\text { Sig }^{6}\end{array}$ \\
\hline \multicolumn{12}{|l|}{$\omega-3$ derivatives } \\
\hline 2-Propenal & 30 & $<500$ & 862 & $\mathrm{~B}$ & $0.48 \pm 0.26$ & $6.42 \pm 2.20$ & $* *$ & $1.34 \pm 0.51$ & $2.28 \pm 0.95$ & $2.19 \pm 0.10$ & ns \\
\hline Butanal & 31 & 600 & 891 & A & $1.19 \pm 0.38$ & $5.00 \pm 0.46$ & $* * *$ & $4.67 \pm 0.50^{\mathrm{a}}$ & $5.96 \pm 0.53^{\mathrm{a}}$ & $11.10 \pm 1.70^{\mathrm{b}}$ & $* * *$ \\
\hline 2-Ethylfuran & 32 & 702 & 970 & A & nd & $2.83 \pm 1.40$ & $* *$ & $0.59 \pm 0.11^{\mathrm{a}}$ & $0.92 \pm 0.15^{\mathrm{a}}$ & $5.31 \pm 1.40^{\mathrm{b}}$ & $* * *$ \\
\hline 1-Penten-3-one & 33 & 687 & 1045 & A & $0.34 \pm 0.13$ & $33.90 \pm 8.70$ & $* * *$ & $1.34 \pm 0.14^{\mathrm{a}}$ & $1.24 \pm 0.09^{\mathrm{a}}$ & $5.45 \pm 0.90^{\mathrm{b}}$ & $* * *$ \\
\hline 2-Butenal (E) & 34 & 650 & 1071 & A & $0.40 \pm 0.19$ & $9.10 \pm 1.70$ & $* * *$ & $1.36 \pm 0.16^{\mathrm{a}}$ & $0.52 \pm 0.09^{b}$ & $2.13 \pm 0.36^{c}$ & $* * *$ \\
\hline 1-Penten-3-ol & 35 & 686 & 1215 & A & $1.67 \pm 0.90$ & $18.90 \pm 6.10$ & $* *$ & $22.30 \pm 2.00^{\mathrm{a}}$ & $13.50 \pm 8.00^{\mathrm{a}}$ & $59.40 \pm 6.60^{\mathrm{b}}$ & $* * *$ \\
\hline 2-Hexenal (E) & 36 & 856 & 1281 & A & nd & $11.50 \pm 3.30$ & $* * *$ & $4.16 \pm 0.59$ & $3.99 \pm 0.44$ & $4.18 \pm 0.74$ & ns \\
\hline 2-(2-Pentenyl)furan (E) & 37 & 1002 & 1330 & A & nd & $3.12 \pm 1.80$ & $* *$ & $\mathrm{nd}^{\mathrm{a}}$ & $0.03 \pm 0.05^{\mathrm{a}}$ & $0.48 \pm 0.09^{b}$ & $* * *$ \\
\hline 2-Penten-1-ol (Z) & 38 & 768 & 1358 & A & $0.32 \pm 0.14$ & $0.95 \pm 0.36$ & $*$ & $0.74 \pm 0.09^{a}$ & $0.94 \pm 0.23^{\mathrm{a}}$ & $4.61 \pm 0.50^{b}$ & $* * *$ \\
\hline 2,4-Heptadienal (E,Z) & 39 & 1004 & 1517 & $\mathrm{~B}$ & $0.49 \pm 0.27$ & $12.30 \pm 3.10$ & $* * *$ & $2.14 \pm 0.13^{\mathrm{a}}$ & $2.11 \pm 0.30^{\mathrm{a}}$ & $3.56 \pm 0.43^{b}$ & $* * *$ \\
\hline 2,4-Heptadienal (E,E) & 310 & 1017 & 1551 & A & $0.79 \pm 0.44$ & $29.70 \pm 7.60$ & $* * *$ & $3.75 \pm 0.46^{\mathrm{a}}$ & $2.94 \pm 0.63^{\mathrm{a}}$ & $4.72 \pm 0.55^{b}$ & $* *$ \\
\hline 3,5-Octadien-2-one (E,E) & 311 & 1074 & 1623 & A & $0.33 \pm 0.36$ & $6.65 \pm 1.90$ & $* * *$ & $0.70 \pm 0.10^{\mathrm{a}}$ & $0.47 \pm 0.19^{a}$ & $2.81 \pm 0.55^{\mathrm{b}}$ & $* * *$ \\
\hline 1-Pentanol & 312 & 769 & 1294 & A & $2.95 \pm 1.30$ & $35.80 \pm 11.00$ & $* * *$ & $46.00 \pm 4.00^{\mathrm{a}}$ & $48.50 \pm 8.90^{\mathrm{a}}$ & $147.0 \pm 21.0^{\mathrm{b}}$ & $* * *$ \\
\hline \multicolumn{12}{|l|}{$\omega-6$ derivatives } \\
\hline Pentanal & 60 & 702 & 997 & $\mathrm{~A}$ & $5.52 \pm 3.73$ & $72.83 \pm 23.53$ & $* *$ & $68.40 \pm 8.18^{a}$ & $77.36 \pm 7.78^{\mathrm{a}}$ & $185.0 \pm 39.0^{\mathrm{b}}$ & $* * *$ \\
\hline Hexanal & 61 & 804 & 1111 & A & $25.94 \pm 27.67$ & $316.0 \pm 92.4$ & $* * *$ & $372.5 \pm 47.7^{\mathrm{a}}$ & $337.3 \pm 80.3^{a}$ & $899.1 \pm 200.6^{b}$ & $* * *$ \\
\hline Heptanal & 62 & 904 & 1240 & A & $7.47 \pm 4.20$ & $26.27 \pm 11.87$ & $*$ & $14.30 \pm 2.10^{\mathrm{a}}$ & $21.60 \pm 4.30^{\mathrm{a}}$ & $36.50 \pm 8.00^{\mathrm{b}}$ & $* * *$ \\
\hline 2-Pentylfuran & 63 & 992 & 1274 & A & $0.84 \pm 0.52$ & $28.43 \pm 16.10$ & $*$ & $1.12 \pm 0.23^{\mathrm{a}}$ & $4.03 \pm 1.40^{\mathrm{a}}$ & $12.64 \pm 1.90^{\mathrm{b}}$ & $* * *$ \\
\hline
\end{tabular}




\begin{tabular}{|c|c|c|c|c|c|c|c|c|c|c|c|}
\hline 2-Heptenal (E) & 64 & 962 & 1380 & A & $14.40 \pm 12.00$ & $136.1 \pm 43.6$ & $* *$ & $19.18 \pm 1.50^{\mathrm{a}}$ & $17.52 \pm 1.40^{\mathrm{a}}$ & $26.54 \pm 4.41^{\mathrm{b}}$ & $* *$ \\
\hline 1-Octen-3-ol & 65 & 982 & 1472 & $\mathrm{~A}$ & $6.42 \pm 3.60$ & $75.47 \pm 28.49$ & $* *$ & $23.26 \pm 3.50^{\mathrm{a}}$ & $30.57 \pm 8.32^{\mathrm{a}}$ & $106.1 \pm 21.0^{\mathrm{b}}$ & $* * *$ \\
\hline 1-Octen-3-one & 66 & 980 & 1350 & $\mathrm{~A}$ & $2.83 \pm 1.30$ & $49.00 \pm 19.00$ & $* *$ & $1.41 \pm 0.20^{\mathrm{a}}$ & $2.46 \pm 1.00^{\mathrm{a}}$ & $8.18 \pm 1.90^{\mathrm{b}}$ & $* * *$ \\
\hline 2-Octenal (E) & 67 & 1061 & 1481 & A & $8.96 \pm 7.00$ & $123.0 \pm 36.0$ & $* * *$ & $8.14 \pm 1.30^{\mathrm{a}}$ & $7.54 \pm 2.50^{\mathrm{a}}$ & $40.90 \pm 4.90^{\mathrm{b}}$ & $* * *$ \\
\hline 3-Octen-2-one & 68 & 1041 & 1458 & $\mathrm{~A}$ & nd & $5.61 \pm 2.00$ & $* *$ & $0.33 \pm 0.12^{\mathrm{a}}$ & $0.16 \pm 0.06^{\mathrm{a}}$ & $3.05 \pm 0.75^{\mathrm{b}}$ & $* * *$ \\
\hline 3-Nonen-2-one & 69 & 1140 & 1554 & $\mathrm{~A}$ & nd & $12.40 \pm 3.00$ & $* * *$ & $\mathrm{nd}^{\mathrm{a}}$ & $\mathrm{nd}^{\mathrm{a}}$ & $0.59 \pm 0.03^{b}$ & $* * *$ \\
\hline 2-Nonenal (E) & 610 & 1163 & 1585 & A & $4.90 \pm 2.80$ & $27.00 \pm 8.00$ & $* *$ & $4.70 \pm 0.54^{\mathrm{a}}$ & $6.05 \pm 0.71^{\mathrm{b}}$ & $6.46 \pm 1.10^{\mathrm{b}}$ & $*$ \\
\hline 2,4-Nonadienal (E,E) & 613 & 1222 & 1755 & A & nd & $3.59 \pm 1.06$ & $* * *$ & $1.74 \pm 0.17^{\mathrm{a}, \mathrm{b}}$ & $1.18 \pm 0.32^{\mathrm{a}}$ & $2.01 \pm 0.48^{b}$ & * \\
\hline 2,4-Decadienal (E,Z) & 614 & 1302 & 1811 & $\mathrm{~B}$ & $0.10 \pm 0.21$ & $44.24 \pm 10.35$ & $* * *$ & $0.87 \pm 0.08^{\mathrm{a}}$ & $0.83 \pm 0.18^{\mathrm{a}}$ & $2.87 \pm 0.49^{b}$ & $* * *$ \\
\hline 2,4-Decadienal (E,E) & 615 & 1324 & 1866 & A & $2.26 \pm 1.56$ & $229.5 \pm 48.0$ & $* * *$ & $3.14 \pm 0.42^{\mathrm{a}}$ & $2.60 \pm 0.68^{\mathrm{a}}$ & $9.61 \pm 1.50^{\mathrm{b}}$ & $* * *$ \\
\hline \multicolumn{12}{|l|}{$\omega-9$ derivatives } \\
\hline 1-Decene & 90 & nd & 1045 & $\mathrm{C}$ & $8.34 \pm 7.10$ & $3.93 \pm 0.93$ & $\mathrm{~ns}$ & $4.00 \pm 4.30^{\mathrm{a}}$ & $17.50 \pm 5.30^{\mathrm{b}}$ & $2.03 \pm 0.30^{\mathrm{a}}$ & $* * *$ \\
\hline 1-Octanol & 94 & 1072 & 1578 & A & $7.58 \pm 2.60$ & $22.30 \pm 6.40$ & $* *$ & $9.90 \pm 0.54^{\mathrm{a}}$ & $13.6 \pm 2.80^{\mathrm{a}}$ & $23.30 \pm 3.60^{\mathrm{b}}$ & $* * *$ \\
\hline 1-Nonanol & 95 & 1172 & 1674 & $\mathrm{~A}$ & $3.73 \pm 2.30$ & $4.76 \pm 1.10$ & $\mathrm{~ns}$ & $2.32 \pm 1.30$ & $4.49 \pm 1.80$ & $2.77 \pm 0.60$ & ns \\
\hline 1-Decanol & 96 & nd & 1773 & $\mathrm{C}$ & $5.32 \pm 3.40$ & $4.22 \pm 2.40$ & $\mathrm{~ns}$ & $3.77 \pm 2.80^{\mathrm{a}, \mathrm{b}}$ & $7.78 \pm 3.60^{\mathrm{a}}$ & $2.10 \pm 0.80^{\mathrm{b}}$ & $*$ \\
\hline 2-Undecenal & 97 & 1367 & 1796 & $\mathrm{~A}$ & $4.93 \pm 2.60$ & $33.90 \pm 7.60$ & $* * *$ & $9.29 \pm 1.10^{\mathrm{a}}$ & $5.83 \pm 1.20^{\mathrm{b}}$ & $6.96 \pm 1.40^{\mathrm{b}}$ & * \\
\hline \multicolumn{12}{|l|}{ Ketones } \\
\hline 2-Pentanone & $\mathrm{k} 1$ & 687 & 996 & $\mathrm{~A}$ & $0.76 \pm 0.15$ & $1.69 \pm 0.19$ & $* * *$ & $10.11 \pm 2.30$ & $18.20 \pm 7.04$ & $15.70 \pm 3.40$ & ns \\
\hline 3-Hexanone & $\mathrm{k} 2$ & 783 & 1082 & A & $0.57 \pm 0.20$ & $1.85 \pm 0.69$ & $*$ & $3.88 \pm 0.73^{\mathrm{a}}$ & $3.68 \pm 1.30^{\mathrm{a}}$ & $0.87 \pm 0.50^{\mathrm{b}}$ & $* *$ \\
\hline
\end{tabular}




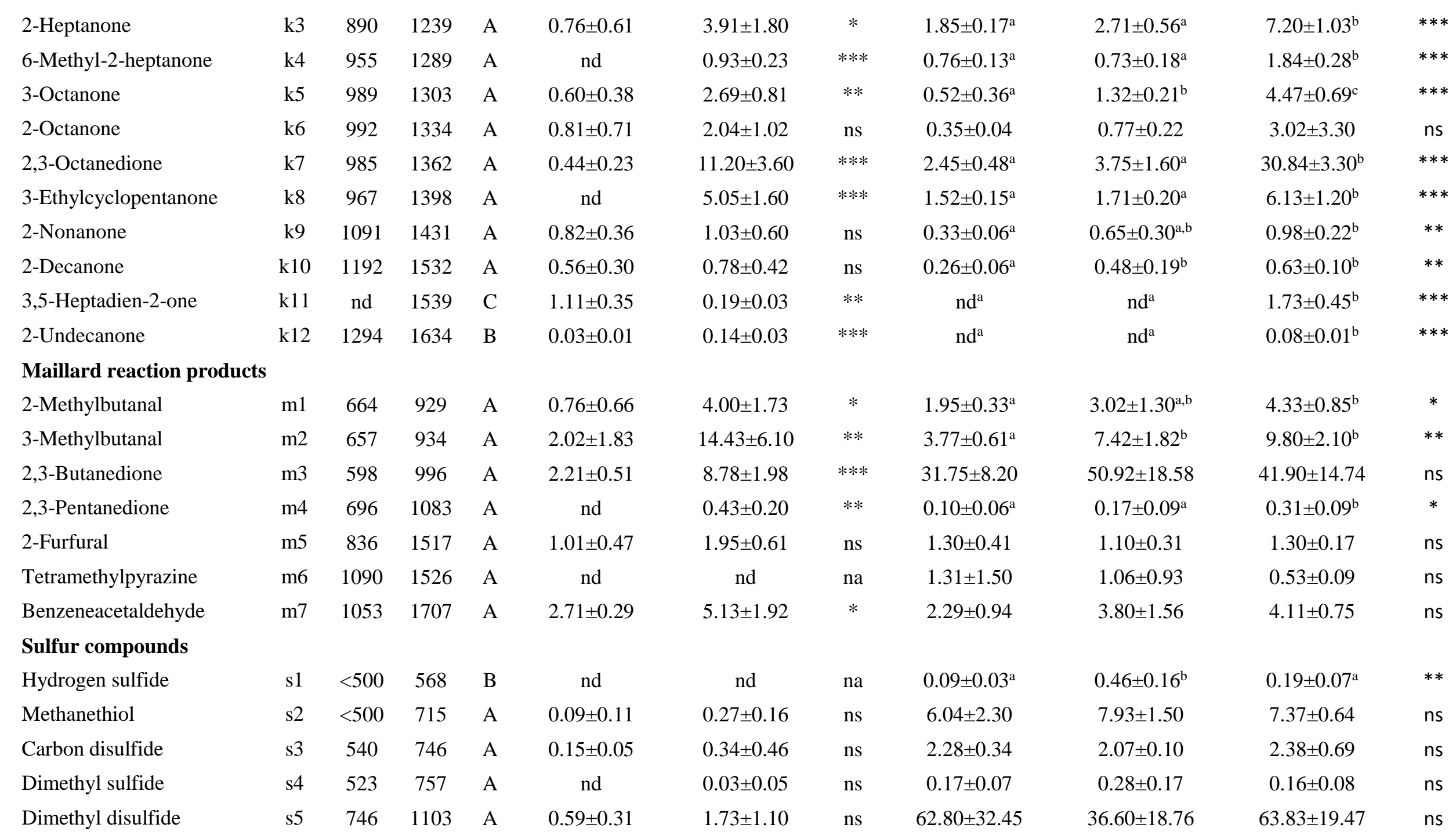




\begin{tabular}{|c|c|c|c|c|c|c|c|c|c|c|c|}
\hline Dimethyl trisulfide & s6 & 977 & 1450 & $\mathrm{~A}$ & $0.28 \pm 0.32$ & $0.27 \pm 0.12$ & $\mathrm{~ns}$ & $55.93 \pm 33.35$ & $44.90 \pm 24.96$ & $52.95 \pm 23.27$ & ns \\
\hline Methional & s7 & 912 & 1517 & $\mathrm{~A}$ & nd & nd & na & $3.07 \pm 1.64$ & $4.98 \pm 1.40$ & $4.14 \pm 0.77$ & ns \\
\hline \multicolumn{12}{|l|}{ Miscellaneous } \\
\hline Nonane & $\mathrm{z} 1$ & 900 & 900 & A & $2.23 \pm 1.1$ & $2.03 \pm 0.55$ & $\mathrm{~ns}$ & $0.69 \pm 0.25^{\mathrm{a}}$ & $4.45 \pm 1.80^{\mathrm{b}}$ & $3.32 \pm 0.38^{\mathrm{b}}$ & $* *$ \\
\hline 1-Hexanol & $\mathrm{z} 2$ & 869 & 1384 & A & $2.35 \pm 0.43$ & $4.79 \pm 1.90$ & $*$ & $7.84 \pm 0.55^{\mathrm{a}}$ & $10.40 \pm 1.50^{\mathrm{a}}$ & $16.90 \pm 2.30^{\mathrm{b}}$ & $* * *$ \\
\hline 1-Tetradecene & $\mathrm{z} 3$ & nd & 1459 & $\mathrm{C}$ & $31.7 \pm 7.5$ & $0.45 \pm 0.52$ & $* * *$ & $0.59 \pm 0.49^{\mathrm{a}}$ & $43.30 \pm 6.40^{\mathrm{b}}$ & $4.62 \pm 1.80^{\mathrm{a}}$ & $* * *$ \\
\hline Undecanal & $\mathrm{z} 4$ & 1309 & 1641 & $\mathrm{~A}$ & $2.12 \pm 0.63$ & $3.58 \pm 1.00$ & $*$ & $1.83 \pm 0.54$ & $1.80 \pm 1.15$ & $2.92 \pm 0.55$ & ns \\
\hline $\begin{array}{l}\text { 6-Methyl-3,5-heptadiene-2- } \\
\text { one }\end{array}$ & $\mathrm{z} 5$ & nd & 1646 & $\mathrm{C}$ & $0.15 \pm 0.02$ & $16.50 \pm 2.80$ & $* * *$ & $\mathrm{nd}^{\mathrm{a}}$ & $0.09 \pm 0.06^{\mathrm{a}}$ & $2.66 \pm 0.23^{b}$ & $* * *$ \\
\hline Dodecanal & z6 & 1410 & 1743 & A & $2.84 \pm 0.57$ & $3.82 \pm 0.58$ & ns & $4.99 \pm 5.70$ & $4.08 \pm 1.70$ & $3.87 \pm 1.10$ & ns \\
\hline
\end{tabular}

${ }^{1}$ Linear retention indices determined on a DB 5 column, nd $=$ not detected.

$456 \quad{ }^{2}$ Linear retention indices determined on a Supelcowax 10 column.

$457{ }^{3}$ Confirmation of identity where $\mathrm{A}=$ mass spectrum and LRI agree with those of an authentic compound; $\mathrm{B}=$ mass spectrum agrees with reference spectrum in the NIST mass spectral database and the LRI value of DB5 agrees with that in the database (NIST Chemistry WebBook,

459 2017); $\mathrm{C}=$ mass spectrum agrees with reference spectrum in the NIST mass spectral database (NIST/EPA/MSDC, 1992). 
${ }^{5}$ Probability, obtained from a T-Ttest that there is a difference between means; ns $=$ no significant difference between means, na $=$ not applicable.

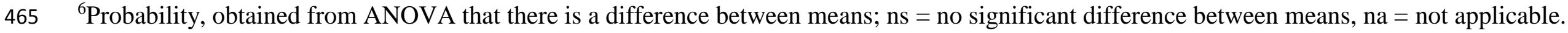


467 Table 2. The content (\%) of unsaturated fatty acids in neutral lipids and phospholipids 468 from chicken meat and hen egg.

\begin{tabular}{lcccc}
\hline Fatty acid* & $\begin{array}{c}\text { Chicken meat } \\
\text { neutral lipids }\end{array}$ & $\begin{array}{c}\text { Chicken meat } \\
\text { phospholipids }\end{array}$ & $\begin{array}{c}\text { Hen egg } \\
\text { neutral lipids }\end{array}$ & $\begin{array}{c}\text { Hen egg } \\
\text { phospholipids }^{\mathrm{b}}\end{array}$ \\
\hline $\mathrm{C} 18: 1$ & 35 & 16 & 53 & 26 \\
$\mathrm{C} 18: 2$ & 25 & 17 & 14.5 & 14 \\
$\mathrm{C} 18: 3$ & 1.3 & 0.5 & 2.1 & 0.5 \\
$\mathrm{C} 20: 4$ & 0.5 & 15 & 0.3 & 7.5 \\
$\mathrm{C} 22: 5$ & 0 & 1.7 & 0.1 & 0.8 \\
$\mathrm{C} 22: 6$ & 0 & 3.9 & 0.3 & 6.5 \\
\hline
\end{tabular}

$469{ }^{*} \mathrm{C} 18: 1$, oleic acid; C18:2, linoleic acid; C18:3, linolenic acid; C20:4, arachidonic 470 acid; C20:5, eicosapentaenoic acid; C22:6, docosahexaenoic acid.

$471 \quad{ }^{a}$ Katz et al., 1966; ' ${ }^{b}$ Fredriksson et al., 2006.

472 


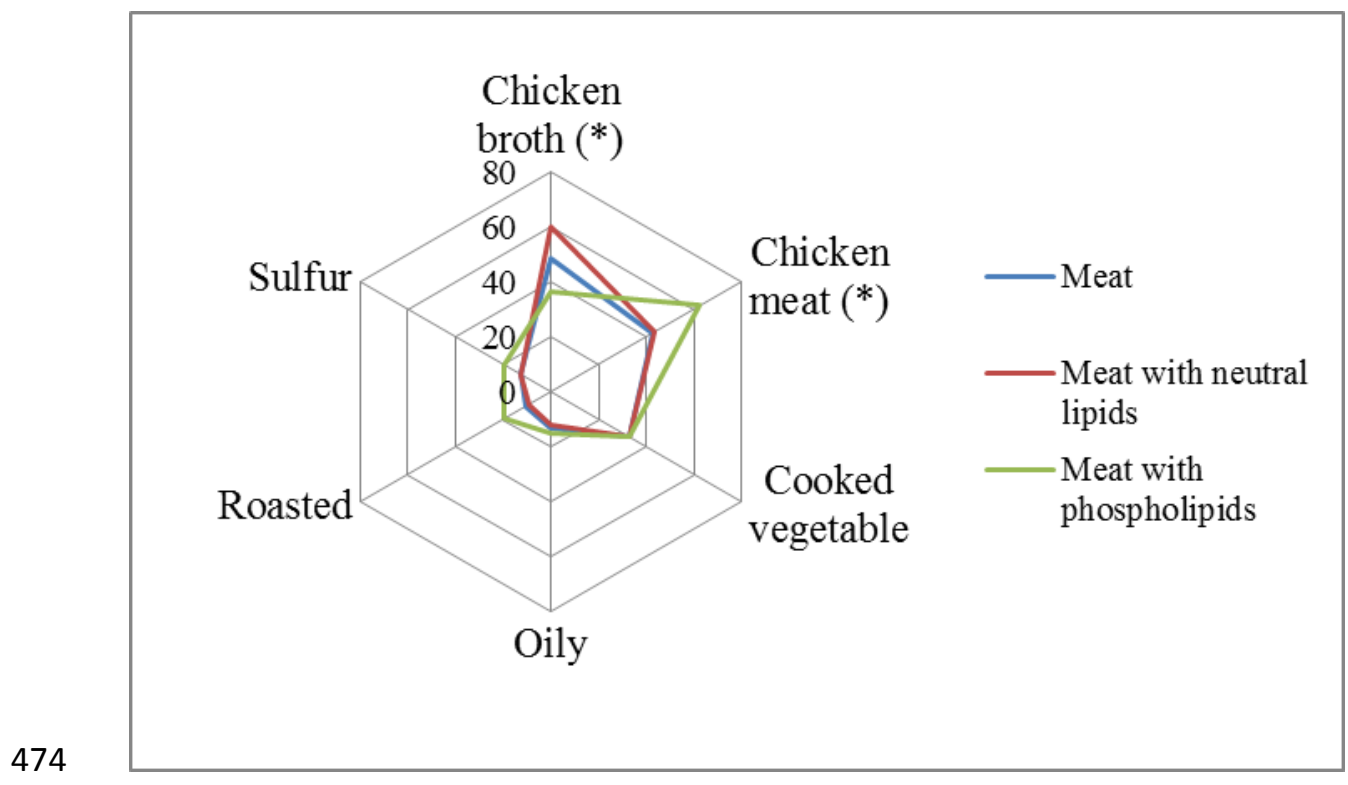

475 Figure 1. Spider diagram of sensory evaluation of the aroma of three chicken meat

476 samples. Mean scores of duplicate analysis $(n=9), *$ indicates significant difference

477 between samples at $\mathrm{p}<0.05$ 


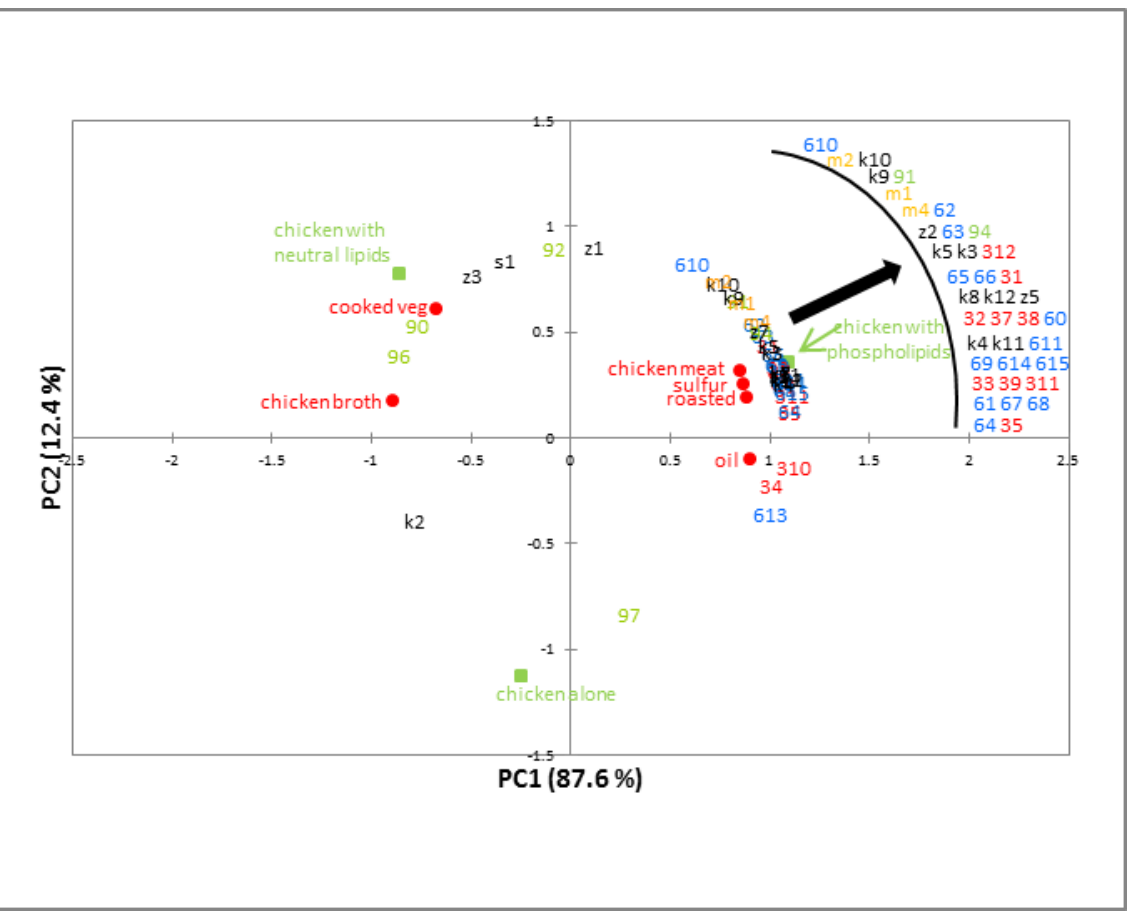

481 Figure 2. Principal component analysis (PC1 vs. PC2) showing sensory data (red) obtained from the chicken samples (green) with the volatile compounds included as supplementary data. Red, blue and green codes are volatiles derived from $\omega-3, \omega-6$ and $\omega-9$ fatty acids respectively, yellow codes are Maillard-derived compounds and the remaining volatiles are black. All codes are defined in Table 1. 